\title{
Diafragma: Trayectoria Histórica del Término y de sus Descripciones Anatómica y Funcional
}

\author{
Diaphragm: Historical Path of the Term and its Anatomical and Functional Descriptions
}

\author{
Rodrigo Muñoz ${ }^{1}$ \& Bélgica Vásquez ${ }^{2}$
}

MUÑOZ, R. \& VÁSQUEZ, B. Diafragma: Trayectoria histórica del término y sus descripciones anatómica y funcional. Int. J. Morphol., 35(4):1614-1622, 2017.

RESUMEN: La historia es una disciplina que permite ordenar un conjunto de conocimientos, legados y realidades históricas complejas del ser humano a lo largo del tiempo y a través de las regiones. Por tanto, conocer la historia de civilizaciones, culturas y sociedades nos permite comprender y racionalizar la información y, posteriormente, tomar esta información para seguir construyendo una nueva realidad. Bajo este contexto, el objetivo de este trabajo fue analizar el origen y significado del término diafragma y, el conocimiento que se tenía de la anatomía y función de éste músculo, para comprender cómo, desde la trayectoria histórica, se construyó su definición actual. En los escritos de Homero, el diafragma fue reconocido como una estructura anatómica que no estaba vinculada a ninguna función en particular y en las primeras explicaciones fisiológicas de la respiración tampoco desempeñaba un rol este proceso. Hipócrates y Platón marcaron un punto de inflexión en la definición del término, ya que lo describieron como una estructura que separaba el tórax del abdomen, ajustándose correctamente con el significado del término diáphragma $(\delta 1 \alpha \phi \rho \alpha \gamma \mu \alpha)$, "separación"; sin embargo, en la época clásica de Grecia fue asociado más frecuentemente a la mitología griega y a la espiritualidad humana considerando al diafragma como sede del pensamiento. Otro hito importante en la historia de este músculo fueron los estudios realizados por Galeno, a través de disecciones en animales y Vesalio en humanos, ya que describen, a partir de observaciones directas, con gran detalle la anatomía del diafragma y se aproximan de manera más adecuada a su función en el proceso de la respiración. Finalmente, Testut estructura y ordena este conocimiento, el cual se ha mantenido hasta la actualidad, en donde sólo ha cambiado la forma en la que se entrega esta información.

PALABRAS CLAVE: Diafragma; Historia; Anatomía; Función.

\section{INTRODUCCIÓN}

La historia de la anatomía y de la medicina muestran que la incorporación y adaptación progresiva de los cultismos al discurso de la anatomía tuvieron varios cambios formales hasta llegar a su completa adaptación. En algunos casos, los médicos de la época, tomaron estos neologismos conservando su forma original, presentándolos de distintas maneras en su voz clásica, hasta alcanzar una forma estable en periodos posteriores. En particular, el término diafragma, sufrió pocos cambios en su transición desde el griego al latín y desde éste al castellano (Pardo \& Martínez, 1997; García, 2010); sin embargo, resulta sorprendente las derivaciones y los significados atribuidos, así como también, las confusiones que surgieron en la historia de la medicina al usar y tratar de denominar con este nombre diferentes áreas del cuerpo y de la mente (Marangoni, 2010).

Diafragma significa separación, proviene del griego, diáphragma $(\delta 1 \alpha \phi \rho \alpha \gamma \mu \alpha)$. Es una palabra compuesta: diá $(\delta 1 \alpha)($ sign. "a través de") + phrág-m(a) $(\phi \rho \alpha \gamma \mu \alpha)$, compuesto del lexema verbal phrag "separar" y el sufijo $m a(\mu \alpha)$ que expresa el resultado de la acción, por tanto "barrera", "separación" (Cortés \& Ureña, 2011). Este término fue utilizado para denominar diferentes partes del cuerpo. Antiguamente a la membrana suprapleural se le denominaba diafragma cervicotorácico. Existen en Terminologia Anatomica otros términos como diafragma pélvico y diafragma urogenital, entre otros.

Cabe mencionar que el término diafragma también es utilizado en los lenguajes coloquial y técnico, para nombrar objetos, como un dispositivo anticonceptivo, parte de una máquina fotográfica, en micrófonos, manómetros, etc. No obstante lo anterior, fue de interés para este estudio abordar el término diafragma aplicado al músculo que separa la cavidad torácica de la cavidad abdominal. Advertir además, que la estructura es nombrada solo como diafragma ya que en Terminologia Anatomica no lleva el prefijo músculo.

\footnotetext{
${ }^{1}$ Programa Doctorado en Ciencias Morfológicas, Facultad de Medicina, Universidad de La Frontera, Chile.

${ }^{2}$ Facultad de Ciencias de la Salud, Universidad de Tarapacá, Chile.
} 
Los médicos a partir de Hipócrates (ss. V-IV a.C.) coincidían en que diáphragma $(\delta i \alpha \phi \rho \alpha \gamma \mu \alpha)$ era sinónimo de phrén ( $\phi \rho \eta v)$, el que se usaba casi siempre en plural, phrénes $(\phi \rho \eta \nu \varsigma)$ cuyo significado era mente, ya que diafragma se asoció con el lugar donde se asientan las pasiones y la inteligencia (Cortés \& Ureña).

La descripción anatómica y las funciones asociadas a este músculo con el tiempo fueron cada vez más complejas, en la cual, los estudios observacionales, de vivisecciones y disecciones tuvieron un rol fundamental en la consolidación actual de su definición.

Por otra parte, se debe considerar que la transmisión de saberes se delimitaba al latín, lengua utilizada en las universidades durante la edad media y siglo XVI hasta inicios del XVII (Micheli-Serra, 2003). Este hecho, favoreció que muchos de los tratados anatómicos antiguos, fueran traducidos al latín y que paralelamente se realizaran modificaciones en las descripciones anatómicas de éstos. Además, el conocimiento del latín permitió a los médicos universitarios la lectura directa de las obras de autores europeos de aquellas épocas, las cuales fueron complementadas con las traducciones y composiciones originales en el idioma vernáculo (García).

En la actualidad, el diafragma se define como un músculo impar que constituye, por sí sólo, la región diafragmática; tiene la forma de una cúpula y su centro es una aponeurosis de donde nacen sus fascículos musculares, que por costal se insertan en el margen inferior del tórax y por posterior, gracias a pilares tendinosos, en las vértebras lumbares.

Standring (2016) señaló que el diafragma aunque sea una lámina continua, puede ser considerado en tres partes, esternal, costal y lumbar, las cuales están basadas en las regiones de inserción periférica. Agrega que las posiciones de las cúpulas del diafragma son extremadamente variables porque ellas dependen de la estructura del cuerpo y de la fase de ventilación. Así, el diafragma estará en posición más alta en personas bajas y obesas que en personas altas y delgadas.

La contracción del diafragma en interacción con la pared torácica, pulmones y abdomen produce la inspiración (Testut, 1899; Feneis \& Dauber, 2000; Standring), acción que permite proveer de aire rico en oxígeno a los distintos tejidos del organismo.

El objetivo de este artículo fue analizar el origen del término y su significado y, el conocimiento que tenían los estudiosos del cuerpo humano de la anatomía y función del diafragma, para comprender cómo, desde la trayectoria histórica, se construyó su definición actual.

\section{MATERIAL Y MÉTODO}

Se efectuó una búsqueda bibliografía, no sistemática, de libros y artículos científicos que describan la anatomía y función del diafragma.

Los textos seleccionados se registraron en una tabla según año de publicación, nombre del autor, referencia de la obra, descripción anatómica y funcional del diafragma.

Para la construcción de la tabla se consideró como autor al investigador que realizó la descripción anatómica y/o funcional del músculo y no a quien cita la información en los libros y artículos científicos los cuales pueden estar o no referenciados en la tabla.

\section{RESULTADOS}

La información fue ordenada cronológicamente según año de publicación de la obra con el fin de facilitar el análisis de la trayectoria histórica de la descripción anatómica y funcional del diafragma (Tabla I).

\section{DISCUSIÓN}

El término diafragma fue acuñado en Grecia en la edad antigua; proviene de una de sus funciones, delimitar el tórax del abdomen. En latín esta estructura corresponde a septum transversum, por tanto, se entiende que este término es de origen griego ( $\delta i \alpha \phi \rho \alpha \gamma \mu \alpha)$, y significa "separación". Posteriormente $\delta 1 \alpha \phi \rho \alpha \gamma \mu \alpha$ fue transliterado al latín antiguo como diáphragma, el cual se mantuvo en el latín medieval y luego derivó a las lenguas romances; por lo qué este término se podría considerar un neologismo heredado que con el tiempo se describió y delimitó para nuestra lengua (García).

Desde el punto de vista mitológico, el término diafragma estuvo entrelazado con los dioses y fue citado en numerosas pasajes por Homero en «La Ilíada» y en relación con el arco iris. En la mitología griega, el arco iris era la trayectoria que realizaba Diafragma, mensajera de los dioses, entre el cielo y la tierra. También era conocida como Iris, diosa del arco iris. En la obra «Tragedia» de Eurípides (484 - 480 a. 4C. - 406 a. C.), Diafragma estuvo junto a la diosa Manía, que personificaba la locura en la trágica escena mitológica de la vida de Heracles, cuando poseído por ella, cometió el atroz crimen contra miembros de su propia 
MUÑOZ, R. \& VÁSQUEZ, B. Diafragma: Trayectoria histórica del término y sus descripciones anatómica y funcional. Int. J. Morphol., 35(4):1614-1622, 2017.

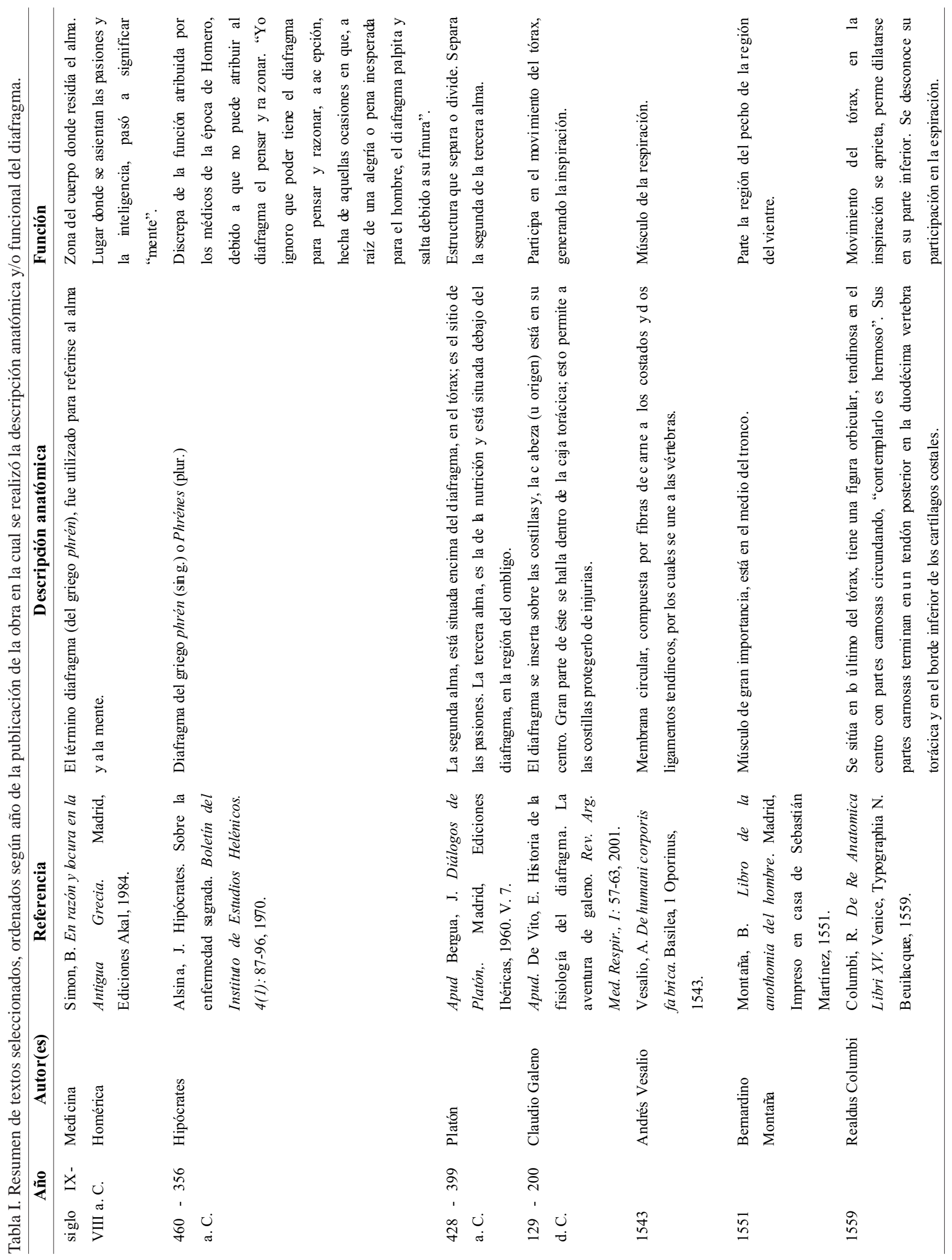


MUÑOZ, R. \& VÁSQUEZ, B. Diafragma: Trayectoria histórica del término y sus descripciones anatómica y funcional. Int. J. Morphol., 35(4):1614-1622, 2017.






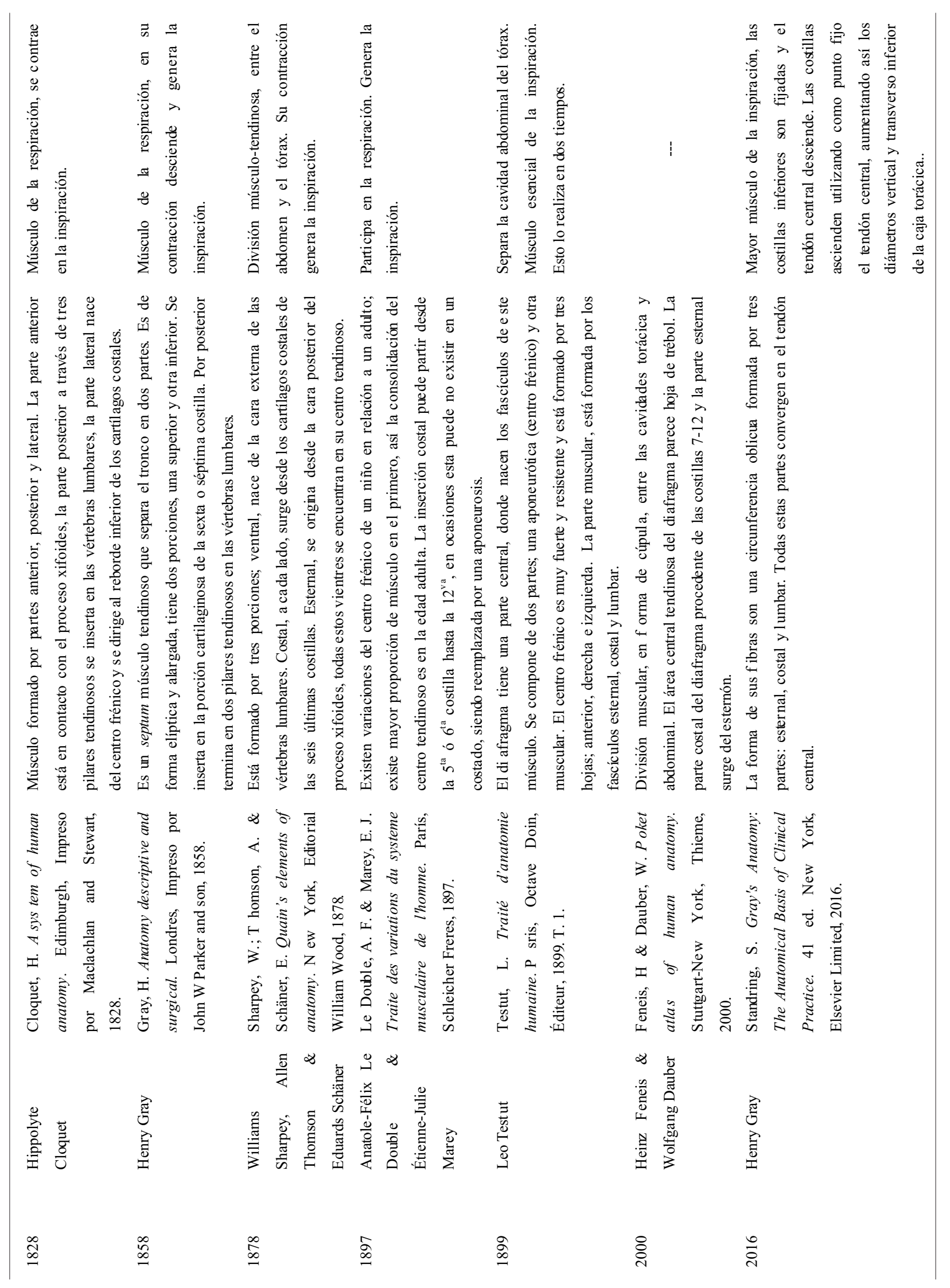


familia y su descendencia. Bajo este contexto, para los griegos clásicos phrén era sinónimo de diáphragma, ya que en un primer momento diáphragma (diafragma) era la sede del miedo y la angustia y, más tarde, por una metonimia phrén (mente), fue la sede del pensamiento (Cortés \& Ureña).

Las obras de Homero en la época clásica de Grecia propiciaron el uso metonímico del término phrén, y se generalizó después de él. Por tanto, el valor habitual de la palabra fuera de los ambientes médicos fue el de "mente". No obstante lo anterior, este significado no fue frecuente en médicos griegos; ejemplo de esto, es el comentario que realizó el autor del tratado hipocrático «Sobre la enfermedad sagrada» quien ponía en claro lo siguiente: "El cerebro es el que articula la intelección. Las phrénes (diafragma) tienen esa denominación (es decir, un término asociado al pensamiento) por azar o convención, pero no por lo que es ni por su naturaleza, porque no sé yo qué facultad tienen para pensar

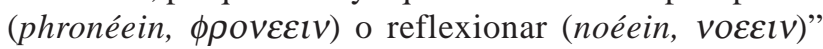
(Alsina, 1970). Por ello, para Hipócrates (460 - 356 a. C.) phrenitis significaba "enfermedad del diafragma" y era un mal que cursaba con fuertes fiebres y muchas veces estaba asociado a la pulmonía por la simple razón de que diafragma y pulmón están cercanos.

Por su parte, Platón (428 - 399 a. C.), también de la época clásica, consideraba al diafragma como una estructura que separa o divide. Según lo señalado en su teoría del alma, el diafragma separaba la segunda alma, ubicada en el tórax, de la tercera alma, ubicada en la región del ombligo (Bergua).

Pese a estas aclaraciones, en la época helénica los lexemas phrén y/o phrénes continuaban utilizándose como "mente". Así, Erasístrato (c. 304 - 250 a. C.), anatomista de la época, afirmaba que phrenitis era una enfermedad cerebral producida por inflamación de las meninges, en consonancia con su idea de que la mente estaba asentada en el cerebro y no en el diafragma. En esta línea, en los siglos XIX y XX se continuó utilizando el lexema phrén para definir enfermedades mentales, entre los cuales se destaca el término Esquizofrenia propuesto en 1911 por el psiquiatra suizo Eugen Bleuler (Marangoni). Esquizofrenia significa división de la mente, proviene del griego Schizophrenie [skhiz(o) $\sigma \chi i \zeta \omega$ (verbo) "dividir" + phrén ( $\phi \rho \eta v)$ "mente" (diafragma) + -ía gr. "cualidad"] (Cortés \& Ureña).

En cuanto a la anatomía y función del diafragma, las obras clásicas analizadas no mostraron descripciones detalladas. Hipócrates y Platón reconocían a este músculo como una estructura anatómica que separaba al tórax del abdomen, sin embargo, ninguno le adjudicó una participación de relevancia en la fisiología de la respiración.
Mayores avances se realizaron en Alejandría, entre los siglos III y IV a. C. Herophilus (340 a. C.), fundador de la anatomía por sistemas; fue el primero en reconocer que los músculos eran los responsables del movimiento. Por su parte, Erasístrato (304 a. C.), a través de estudios experimentales en animales, demostró que los músculos respiratorios eran las estructuras que generaban el movimiento respiratorio. Esto abrió el camino a los descubrimientos posteriores realizados por Galeno (129 - 200 d. C.) en la Edad Media (De Vito, 2001).

Galeno, fue un prestigioso médico griego que vivió en Roma a partir del año 162 d. C. en donde escribió numerosas obras, demostrando públicamente su conocimiento de la anatomía. Galeno describió el diafragma de manera más rigurosa a través de la observación directa en disecciones y vivisecciones en animales experimentales y a través de estudios clínicos. Describió con detalle la pleura, los orígenes del diafragma en las costillas y en la columna, además del ligamento arcuato, la región crural y su tendón central (De Vito). Según Derenne et al. (1995), para abordar la función del diafragma Galeno realizó una serie de experimentos en animales que le permitieron determinar que éste músculo aumentaba el diámetro inferior del tórax y mantenía la inspiración, demostrando que era el principal músculo en esta función.

Desde Galeno en adelante la literatura mostró mayor detalle en la descripción anatómica del diafragma; esto, probablemente, debido a la gran influencia que tuvo la metodología galénica para el estudio de la medicina por parte de científicos de la edad media. Junto con lo anterior, se observó que el periodo crítico para la descripción actual de este músculo fue el Renacimiento. En esta etapa el humanismo médico se originó en Italia debido a que los grandes médicos de la época eran italianos o desempeñaron sus tareas allí, donde por proximidad esta influencia pasó directamente a España, quien, hasta el último tercio del siglo XVI, participó plenamente en las corrientes científicas propias del Renacimiento (Michelli-Serra, 2003).

Siguiendo la línea de tiempo de la información analizada, aparece la figura de Andrés Vesalio. En 1543 publica el primer tratado moderno de anatomía humana, De humani corporis fabrica, con información clara y rigurosa; sus descripciones provenían del cuerpo humano y no del animal, como las realizadas por Galeno. Se formó como médico en la Universidad de París y Padua; aquí estudió las teorías de Galeno y también nació su interés por la anatomía. Una vez catedrático, Vesalio creía que la observación directa era la única fuente fiable de información, al punto de realizar él mismo sus disecciones, con esto generó un importante quiebre en la enseñanza medieval, la cual se basaba principal- 
mente en textos. En 1539 logró que un juez de Padua, interesado en sus trabajos le facilitase el acceso a cadáveres de los criminales ejecutados para la disección, lo cual le permitió mejorar sus diagramas anatómicos (Romero, 2007). No obstante lo anterior, las descripciones anatómicas del diafragma que realizó Vesalio en su obra De humani corporis fabrica, no diferían mayormente de las descripciones que realizó Galeno en el libro «Procedimientos anatómicos» (Singer, 1956); por ejemplo, ambas obras señalaban que el diafragma se insertaba por anterior en el reborde costal y proceso xifoides $\mathrm{y}$, por posterior, a través de los pilares tendinosos, en la columna lumbar.

A modo de resumen podemos señalar que en el Renacimiento se encontraba establecido claramente que el diafragma cumplía con la función básica de dividir el tórax del abdomen y que además, participaba en la fisiología de la respiración; existiendo mayor consenso en los textos analizados en relación a su participación en la inspiración. Realdus Columbi señaló que el diafragma podría tener algún tipo de trabajo en la espiración, fundamentando lo señalado a partir de observaciones en tórax abierto. Señaló que cuando el tórax es penetrado y se pierde presión pleural, los pulmones se colapsan y el diafragma se contrae; esto lo llevó a pensar que la presión unía el diafragma a los pulmones y que, por tanto, podría regular la espiración con el objetivo de prevenir dicho colapso pulmonar (Columbi, 1559), sin embargo, este fenómeno solo quedo en la hipotetización y no fue comprobado.

El contacto fluido y estratégico entre Italia y España en el siglo XVIII, permitió el desarrollo de la anatomía en este país. Así, Felipe $\mathrm{V}$ propició la llegada de científicos y especialistas desde distintas universidades de Europa con el propósito de actualizar la ciencia en España. En este tránsito, Manuel de Porras en 1716, cirujano de su Majestad y de los Reales Hospitales de la Corte, había publicado su obra «Anatomía galénico-moderna» dirigida a cirujanos; ésta constaba de una descripción detallada de las estructuras del cuerpo humano y los componentes más importantes de su fisiología. Uno de sus grandes aportes fue reunir la anatomía galénica con los desarrollos más recientes de la investigación anatómica de las principales universidades europeas desde finales del siglo XVI (Pardo \& Martínez).

Por su parte, Martín Martínez autodefinido como crítico, antiaristotélico y escéptico, fue médico de cámara de Felipe V, del Hospital General de Madrid y presidente de la Regia Sociedad de Medicina de Sevilla. Publicó en 1728 su libro «Anatomía completa del Hombre», escrito influyente en la época y a lo largo del siglo XVIII. Este cirujano fue conocido como uno de los renovadores de la medicina española y en el ámbito de la terminología estaba en contra del galicismo y cultismo. Por esto, Martínez criticaba constantemente la obra de Manuel De Porras (Pardo \& Martínez). Pese a estas diferencias, para las descripciones del diafragma, ambos se basaron en relatos de lo observado a medida que se ejecutaba la disección desde el exterior al interior de la caja torácica, coincidiendo así con su descripción anatómica y su función, la inspiración.

Más de un siglo después, una vez concordada la información que existía del diafragma con sus observaciones en diversas especies, Le Double, profesor de anatomía de la escuela de anatomía del Tours Lauréat de L'Institut, y Marey, miembro de L'Académie des sciences de L'Académie de médecine y profesor del Cóllege de France, se percataron de cambios morfológicos en los distintos músculos o grupos musculares. En tal escenario, se propusieron detallar las variaciones musculares, para contribuir en la descripción completa de la miología del hombre y con esto facilitar y resolver problemas de orden quirúrgico (Le Double \& Marey, 1897). Estos autores, señalaron que existen variaciones en el diafragma (Tabla I) consideradas como comunes, las cuales no necesitarían de una eventual intervención. En este mismo sentido Sperino apud Testut \& Latarjet (1969) describieron en la cara inferior del diafragma un fascículo, en parte carnoso y en parte tendinoso que se extendía de la parte esternal de la" hojilla media a la hojilla izquierda". Otros como Knox apud Testut \& Latarjet denominó músculo hepaticodiafragmático a un fascículo supernumerario que se extendía de la mitad izquierda del centro tendíneo, cruzaba el esófago e iba a terminar por dos fascículos distintos en el peritoneo, por delante del pilar derecho y en la cara inferior del hígado, a nivel del conducto venoso.

En este periodo, también se destacó el anatomista Jean Leo Testut. Su obra es inmensa, con más de 90 publicaciones en materia de anatomía, antropología, pre-historia e historia. En 1887 publicó el «Tratado de Anatomía Humana» en cuatro volúmenes, con su segunda edición en 1893 , el cual se le considera uno de los tratados de anatomía humana más completos y con una ilustración muy detallada. Esta obra fue laureada por la Academia de Medicina de París (Premio Saintour, 1902); en 1905, presentó en colaboración con Jacob y Billet el «Atlas de disección por regiones»; posteriormente Leo Testut publicó en conjunto con André Latarjet el Compendio de Anatomía Descriptiva.

En la novena edición del «Tratado de Anatomía Humana» de Testut \& Latarjet, revisada, corregida y aumentada con la colaboración de Michel Latarjet, el diafragma, lo ubica en la región diafragmática y la información es desarrollada en seis puntos: i) inserciones (origen e inserción), ii) orificios diafragmáticos, iii) relaciones y iv) inervación, v) vasos y vi) acción. En lo esencial, las descripciones no 
difieren en demasía de las realizadas en el siglo XIX, sin embargo, se denota el orden propuesto por Testut en el trato de la información. Otro punto a destacar, es la participación de Testut en la unificación del lenguaje anatómico, donde fue participante activo al cooperar en la confección de Nomina Anatomica de Basilea (Sakai, 2007), por lo que podríamos pensar que sus descripciones tendrían un lenguaje universal.

Por último, en textos más recientes, como los de Feneis \& Dauber (2000) y Standring, se observó que la información entregada era similar a la desarrollada por Gray (1858), Sharpey et al., (1878), Le Double \& Marey (1897) y Testut (1899); sólo existe una propuesta distinta en la presentación de la información. Así Standring realiza una integración de todos los conocimientos, con el objetivo de crear un relato funcional del diafragma.

\section{CONCLUSIONES}

Podemos señalar que la información presentada permitió distinguir que Hipócrates y Platón marcaron un punto de inflexión en la definición del término, la anatomía y función del diafragma en la época clásica de Grecia, en el cual estos aspectos fueron asociados a la mitología griega y a la espiritualidad humana.

En la Edad Media, se destacaron las obras de Galeno y Vesalio por describir con mayor detalle la anatomía y función del diafragma que se tenía hasta ese momento. Por otra parte, se impulsó la enseñanza de la anatomía en Europa y la traducción de textos a idiomas vernáculos, permitiéndose mayor difusión de la información.

Con la información obtenida a la fecha, anatomistas como Le Double \& Marey realizaron descripciones de las variaciones anatómicas del diafragma, contribuyendo con información relevante para un mejor manejo en los procedimientos quirúrgicos y en aplicaciones clínicas.

Por último, Testut estructura y ordena este conocimiento, el cual se ha mantenido hasta la actualidad, en donde sólo ha cambiado la forma en la que se entrega de la información.

MUÑOZ, R. \& VÁSQUEZ, B. Diaphragm: Historical path of the term and its anatomical and functional descriptions. Int. J. Morphol., 35(4):1614-1622, 2017.

SUMMARY: Over time and throughout all regions, history has been a discipline allowing an established order of knowledge, legacies and complex historical experiences of human beings.
Consequently, knowing the history of civilizations, cultures and societies allows us to understand and rationalize this information and use the information to continue building a new reality. In this context, the objective of this work was to analyze the origin and meaning of the term diaphragm, and knowledge of the anatomy and functions of this muscle. Based on this knowledge and its path throughout history its current definition was constructed. In Homer's writings, the diaphragm was recognized as an anatomical structure that was not linked to any particular function and in the early physiological explanations of breathing, and did not play a role in the breathing process. Hippocrates and Plato marked a point of inflection in the definition of the term, since they described it as a structure that separated the thorax from the abdomen, relating it correctly with the meaning of the term diáphragma (diafragma), "separation". However, in the classical period of Greece, it was more frequently associated with Greek mythology and human spirituality, considering the diaphragm as the seat of thought. Another important milestone in the history of this muscle were the studies Galen through dissection in animals, and Vesalius in humans, where both describe the diaphragm and its functions in great detail, approaching the detail of the breathing process more closely. Finally, Testut structures the information in a manner that has been maintained to this day, and the only change has been in the dissemination of the information.

Function.

KEY WORDS: Diaphragm; History; Anatomy;

\section{REFERENCIAS BIBLIOGRÁFICAS}

Alsina, J. Hipócrates. Sobre la enfermedad sagrada. Boletín del Instituto de Estudios Helénicos. 4(1):87-96, 1970.

Bell, J. \& Bell, C. The anatomy of the human body. $3^{\mathrm{a}}$ ed. London, En la imprenta de A. Straham, 1802.

Bergua, J. Diálogos de Platón. Madrid, Ediciones Ibéricas, 1960. V. 7.

Calvi, M. Tratado del amor y la hermosura. Milan, Editorial de Paulo Gotardo, 1576.

Cheselden, W. The anatomy of the human body. London, Thev editon and Forty Copper Plates, 1711.

Cloquet, H. A system of human anatomy. Edimburgh, Impreso por Maclachlan and Stewart, 1828.

Columbi, R. De Re Anatomica Libri XV. Venice, Typographia N. Beuilacquæ, 1559.

Cortés, F. \& Ureña, J. Diccionario Médico-Biológico, Histórico y Etimológico. Salamanca, Ediciones Universidad de Salamanca, 2011. Disponible en: http://dicciomed. eusal.es.

Da Vigo, J. Libro de práctica en cirugía del muy famoso y experto doctor Juan de Vigo. Madrid, Impreso en casa de Phelippe de Lunta, 1564.

De Porras, M. Anatomía Galénico-Moderna. Madrid, En la imprenta de la Música, por Bernardo Peralta, 1716.

Derenne, J. P.; Debru, A.; Grassino, A. E. \& Whitelaw, W. A. History of diaphragm physiology: the achievements of Galen. Eur. Respir. J., 8(1): 154-60, 1995.

De Vito, E. Historia de la fisiología del diafragma. La aventura de galeno. Rev. Arg. Med. Respir., 1:57-63, 2001.

Feneis, H. \& Dauber, W. Poket atlas of human anatomy. Stuttgart-New York, Thieme, 2000.

García, C. La formación de la terminología anatómica en español (14931604). Tesis doctoral, Universidad de Salamanca 2010. 
Gibson, T, H. The anatomy of humane bodies epitomized. London, Printed for M. Flesher, 1682.

Gray, H. Anatomy descriptive and surgical. Londres, Impreso por John W Parker and son, 1858.

Hervás, L. El Hombre Físico o Anatomía Humana Físico-Filosófica. Madrid, Imprenta de la administración del Real Arbitrio de Beneficencia, 1800 .

Le Double, A. F. \& Marey, E. J. Traité des variations du systeme musculaire de l'homme. París, Schleicher Freres, 1897.

Marangoni, A. Etimología de las palabras frénico y diafragma. Rev. Argent. Radiol. 74(3):251-4, 2010.

Martínez, M. Anatomía completa del Hombre. Madrid, En la imprenta de Bernardo Peralta, 1728.

Montaña, B. Libro de la anothomia del Hombre. Madrid, Impreso en casa de Sebastián Martínez, 1551.

Micheli-Serra, A. Historia y filosofía de la medicina en torno a los orígenes de la ciencia moderna. Gac. Méd. Méx., 139(5):513-7, 2003.

Pardo, J. \& Martínez, A. Los origines del teatro anatómico en Madrid. Asclepio, 49(1):5-38, 1997.

Romero, R. Andreas Vesalius (1514-1564). Fundador de la Anatomía Humana Moderna. Int. J. Morphol., 25(4):847-50, 2007.

Romero, R. Erasistratus of Ceos (310-250 A.C.). Pioneer of the Anatomical Studies. Int. J. Morphol., 26(4):823-4, 2008.

Sakai, T. Historical evolution of anatomical terminology from ancient to modern. Anat. Science Int., 82:65-81, 2007.

Sharpey, W.; Thomson, A. \& Schäner, E. Quain's elements of anatomy. New York, Editorial William Wood, 1878.

Simon, B. En Razón y locura en la antigua Grecia. Madrid, Ediciones Akal, 1984.

Singer, C. Galen on Anatomical Procedures. Book V, Chapters 5 and 8. London, Oxford University Press, 1956.

Standring, S. Gray's Anatomy: The Anatomical Basis of Clinical Practice. 41 st ed. New York, Elsevier Limited, 2016.

Testut, L. Traité d`anatomie humaine. 4 éd. Paris, Octave Doin, 1899. Tomo 1.

Testut, L. \& Latarjet, A. Tratado de Anatomía Humana. 9ª ed. Barcelona, Salvat, 1969.

Vesalio, A. De humani corporis fabrica. Basilea, 1. Oporinus, 1543.

\section{Dirección para correspondencia: Dra. Bélgica Vásquez Pastene Facultad de Ciencias de la Salud Universidad de Tarapacá \\ Arica - CHILE}

\section{Email: bvasquezp@uta.cl}

Recibido : 10-09-2017

Aceptado: 08-10-2017 